\title{
Adherence to Medication During Transition to Adult Services
}

\author{
Bianca R. Campagna ${ }^{1,2} \cdot$ Kristen Weatherley $^{2} \cdot$ Eyal Shemesh $^{2} \cdot$ Rachel A. Annunziato $^{1,2}$ (])
}

Published online: 5 September 2020

(c) Springer Nature Switzerland AG 2020

\begin{abstract}
The transition from childhood and adolescence to adulthood is often tumultuous. For individuals with a chronic medical condition, this progression also includes a gradual transition to independence in healthcare management as well as a transfer in care location at some set point. As adolescents navigate these sometimes challenging processes, there is a significant risk for a decline in adequate health behaviors, which can have dire consequences. One of the most vital components of the transfer to adult care is medication adherence. Poor medication adherence puts patients at risk for worse outcomes, with the most profound being increased mortality for many conditions. In recent years, acknowledgment of the need to create evidencebased methods to aid patients during the transition period has been growing. This paper seeks to provide an overview of current research and recommendations for interventions to increase adherence to medication regimens during this period.
\end{abstract}

\section{Key Points}

The transfer from pediatric to adult care may put some patients at a higher risk for medication nonadherence.

Transition planning for adult care should start early and include both patients and caregivers. Important components of successful interventions likely include those that address educational and psychosocial needs, use objective measures to assess adherence, and provide a point of contact to aid in the transition.

More research is needed to assess the effectiveness of intervention strategies.

Rachel A. Annunziato

annunziato@fordham.edu

1 Department of Psychology, Fordham University, 441 E. Fordham Road, Bronx, NY 10458, USA

2 Department of Pediatrics, Icahn School of Medicine at Mount Sinai, Kravis Children's Hospital, New York, NY, USA

\section{Introduction}

The transition from childhood and adolescence to adulthood is often tumultuous. For individuals with a chronic medical condition, this progression also often includes a gradual transition to independence in healthcare management as well as a transfer in care location at some set point. The transfer to adult care typically takes place between the ages of 18-21 years [1]. During this time, patients are tasked with taking greater responsibility for their health and, at the same time, are transferring to a new set of medical providers, with whom they often have no relationship and who may have different expectations regarding patients' ability to self-manage. Thus, this period is one of the most vulnerable for a decline in adequate health behaviors, sometimes with dire consequences [1].

A review of qualitative research on transfer-of-care experiences identified four major themes: changes in relationships, moving to an unknown culture of care, preparedness for the transfer, and achieving responsibility [2]. In addition to these themes, patients may face barriers related to changing insurance coverage, a lack of adequate healthcare knowledge, and physician mistrust [3-5]. Troublingly, medical providers often do not talk about this transition process [6]. A large survey of adolescents aged 15-17 years with special healthcare needs indicated that only $23 \%$ were receiving transition planning guidance [6]. However, the American Academy of Pediatrics recommends that providers start 
addressing both transition and transfer by early adolescence [1].

One of the most vital health behaviors in individuals with chronic medical conditions is medication adherence. Increased rates of nonadherence to medications have been observed during the transition to self-managed care as well as after transfer between pediatric and adult services [7-9]; this trajectory is further associated with poorer clinical outcomes and increased mortality $[1,9]$. In recent years, there has been a growing acknowledgment of the need for evidence-based methods to aid patients during these processes. Prior comprehensive reviews on this topic can be found in the literature (e.g. Gabriel et al. [10] and Campbell et al. [11]). This paper seeks to add to this literature by providing a succinct overview of current research and recommendations for interventions to specifically increase adherence to medication regimens during this period. We first provide considerations for assessment, including measuring adherence and transition readiness, then we present the relevance of mental health to adherence and transition. Finally, we offer a synthesis of empirical data on interventions that address adherence during transition.

\section{Measuring Adherence}

A critical component of assessing and addressing adherence in both clinical and research settings is the use of accurate, objective adherence measures [12]. Self-reported adherence does not always translate into actual adherence. One review of studies on medication adherence in transplant recipients highlighted those that found "increased adherence" but no improvement in rejection outcomes [12]. It is thus imperative that both clinicians and researchers assess adherence using objective measures rather than those that are easily subject to misreporting. For example, the Medication Level Variability Index (MLVI) has been well-established as a valid approach to measuring adherence in transplant patients across the lifespan [13]. Additionally, adherence may change throughout treatment, even if patients were adherent at baseline [14]. It is vital that adherence continues to be measured throughout the treatment process [14]. We present this research with this caveat and have noted when studies did not incorporate objective measures of adherence.

\section{Interventions in Adherence}

\subsection{Transition Readiness}

One component of a successful transfer from pediatric to adult care may be the readiness of adolescents/young adults for this transfer. Transition readiness includes both the self-efficacy and the ability of adolescents/young adults to take on a more significant role in their own healthcare. Studies on the role of transition readiness and interventions to increase it have produced mixed results in terms of actual increased or maintained adherence [15-18]. The role of transition readiness in the self-reported health outcomes of medication adherence and emergency room visits was examined in a sample of patients with chronic kidney disease; transition readiness was significantly associated with increased self-reported medication adherence and fewer emergency room visits [15]. Additionally, family cohesion was a significant predictor of transition readiness, which may highlight the critical role family members can play in the transfer to adult care [15].

Another study looked at the role of transition readiness in young adults with inflammatory bowel disease (IBD) [18]. Patients completed a transition readiness questionnaire, which included a medication management scale; lower scores on this scale were associated with greater medication and visit nonadherence, as captured by medical records [18]. Yet, findings have been equivocal, and the relationship between transition readiness and adherence outcomes may be complicated $[17,19,20]$ and related to poorly studied assessments [21].

Research in populations with pediatric liver transplants, asthma, and IBD have reported conflicting results [17, 19, 20]. One study looked at the role of transition readiness, specifically readiness to talk with providers and manage daily activities, amongst youth with asthma [19]. These behaviors, as well as perceptions of independence in taking medication, were not related to actual medication adherence. Of note, the outcome variable of this study was self-reported medication nonadherence, which may have been susceptible to misreporting. Interestingly, teenagers who were "ready" were more likely to be white and have a caregiver who was educated past high school, highlighting the potential role of socioeconomic status [19]. Verma and Rohan [20], who studied resilience and transition readiness among pediatric patients with various chronic illnesses, presented similar findings, reporting that neither transition readiness nor resilience was associated with self-reported or actual adherence, as measured by prescription refill data [20].

The potentially nuanced association between transition readiness and adherence has been explored in the pediatric liver transplant context [17, 21]. For example, perceived transition readiness was associated with age but not with medication adherence [17]. Older adolescents and young adults had higher perceived transition readiness but lower medication adherence. In understanding these results, the authors pointed to the difference between perceived and actual skills in disease management [17]. This is an important distinction that may help elucidate the lack of consistent findings in this area. Our group conducted analyses from 
a large sample of pediatric transplant recipients, utilizing the MLVI as an objective measure of medication adherence, and highlighted the importance of caregiver report and their involvement in self-management acquisition [21]. Nonadherence to medication was significantly associated with discrepancies between patient and caregiver reports of selfmanagement and with patient perception of high levels of self-management. We further explored whether this pattern persisted in predicting rejection, an endpoint of nonadherence in this population, and findings were robust.

Finally, emphasis on transition readiness by providers may be disconnected from the actual skills needed for a successful transition [16]. Fishman et al. [16] assessed whether dedicated educational programming for providers, which included at least two 60-min sessions, was associated with the self-management skills of patients with IBD. Patients completed self-report questionnaires, one group before providers received any programming regarding transition readiness, and one after. Though its design limited this study, provider awareness did not impact self-management skills [16]. A possible interpretation of the overall findings in this area is that self-efficacy and actual skills are both required for effective transfer to adult models of care. Interventions must focus on both to be effective.

\subsection{Mental Health}

Mental health is a frequently overlooked factor in medication adherence and may well influence self-efficacy and consistency in applying self-management skills. In the general population, the highest rates of mental illness are seen during the transition from adolescence to adulthood [22]. Data show that young adults have the highest prevalence of a diagnosis of a mental illness in the previous year relative to other age groups, and up to $50 \%$ of young adults may experience diagnosable symptoms [22, 23]. Specifically, today's young adults are facing unprecedented levels of stress and rising rates of anxiety and mood disorders [22]. Psychological wellbeing plays an essential role in medical and health outcomes [24]. To this point, a seminal study [24] found that patients with depression had three times greater odds of not adhering to medical treatment.

To date, data on the interrelationships between mental health and adherence during transition are sparse. A longitudinal study of 20 liver transplant recipients found that psychological distress and medication nonadherence after the transfer to adult care were significantly correlated [25]. Patients who were shown to be nonadherent, using the MLVI, had increased levels of psychological distress [25]. Furthermore, a recent literature review of adherence factors found that, generally, most studies have found mental health to be a significant factor [26]. Given the likely role of mental health in adherence during the transfer period, mental health and risk factors should be assessed and addressed throughout the transfer process.

An important area for further consideration is whether posttraumatic stress may be a factor in poor outcomes during transition. Posttraumatic stress symptoms and posttraumatic stress disorder have emerged as risk factors for nonadherence in populations with chronic medical needs [27-31]. Indeed, recent work utilizing a representative sample of the general population demonstrated the profound impact of trauma exposure on functional impairment in those who experienced a traumatic event before the age of 18 years [32]. In the context of special healthcare needs, the avoidance dimension of posttraumatic stress symptoms has been invoked as a potential mechanistic explanation for the reported association between posttraumatic stress symptoms and nonadherence [28, 29, 31]. Such nonadherence can lead to worsening medical outcomes and complications that, in turn, may lead to re-traumatization and further avoidance [33, 34]. In patients who experience symptoms related to posttraumatic stress, evidence-based interventions, such as prolonged exposure therapy, may serve to both decrease symptoms and increase adherence [31, 34].

\subsection{Role of Families}

Despite the growing independence of adolescents and young adults, families still play a role in the development of successful adherence interventions [35]. Iskander et al. [36] looked at the role of family communication in adherence and glycemic control in patients with type 1 diabetes mellitus over a 3-year period starting from ages 9-11 years. They found that family communication at baseline, but not over time, was a significant predictor of later adherence and glycemic control [36]. Taking this into account, interventions in parent and child communication at early ages may be an important factor in effective adherence interventions.

Few interventions have focused directly on utilizing the family system for improving adherence during this period [37]. However, those that have been published provide promising results. Wysocki et al. [38] utilized diabetes-specific behavioral family systems therapy for adolescents and found that the intervention was effective in improving mean glycemic control [glycated hemoglobin (HbA1c)] but not selfreported adherence, which instead may have mediated this relationship [38]. Another small study, by Duncan et al. [39], assessed the use of the Teamwork Intervention in children with asthma aged 9-15 years and their parents. The Teamwork Intervention focused on effective shared responsibility of asthma management between parents and children, with the child assuming increased age-appropriate independence. The intervention was significantly more effective at increasing adherence and lowering functional severity than were standard care and an educational intervention. Adherence 
was measured using an electronic recording device attached to the inhaler [39]. It is likely that future interventions utilizing the family system will also be successful.

\subsection{Transfer Clinic Programs}

In the past decade, more data have amassed regarding transitional interventions. An important aspect of transition planning is the development of interventions that operationalize transfer as a process, rather than an abrupt event, with stable adherence as one of the objective targets. This understanding may translate into interventions such as the implementation of a transition coordinator or navigator or a transition clinic. The purpose is to provide young people with the guidance and consistency necessary to help them navigate the transfer.

Some studies have looked at the use of transfer clinics to help patients plan for adult care. Cole et al. [40] used a record review to investigate the outcomes of patients with IBD who had gone through a transfer clinic versus those who had not. The transfer clinic included joint evaluations by both adult and pediatric providers, assessments of functioning and readiness for transfer, and a visit from a psychologist and dietician if necessary. Medication adherence was significantly higher in the group that had attended the transfer clinic, as measured by self-reported adherence during clinic visits and significantly lower rates of surgery and hospitalizations [40]. This study gives support to a transfer clinic type of intervention.

Another study assessed the use of a transfer clinic in kidney transplant patients using a retrospective chart review before and after the institution implemented the transfer clinic and reported similar results [41]. The transfer clinic involved visits with a small group of patients, often with a parent. Patients met with adult providers, participated in group discussions centered on adult healthcare and future planning (e.g., education, finances), and attended a graduation celebration. A case review was also conducted for each patient. As part of the intervention, families worked with a coordinator and a single adult nephrologist after the transfer. Patients who were not a part of the transfer clinic had significantly lower rates of adherence, as measured by self-report, missing appointments, and routine blood levels in the year following the transfer [41].

An implementation of a transition clinic in patients with type 1 diabetes mellitus yielded promising results [42]. The clinic consisted of care coordination, orientation to adult care delivered by the diabetes nurse after the first adult appointment, referrals to a dietician and diabetes educator, referrals to psychiatric services if necessary, efforts to increase engagement in care, and increased communication between pediatric and adult providers. Improvements from baseline to 6 months in $\mathrm{HbA} 1 \mathrm{c}$ and blood glucose monitoring frequency were significant, independent of sex, diabetes duration, insulin regimen, and baseline $\mathrm{HbA} 1 \mathrm{c}$ and blood glucose monitoring frequency. This study supports the use of this type of intervention in individuals with type 1 diabetes mellitus [42].

Others have tested the use of a transition program for adults with juvenile idiopathic arthritis [43]. The program included a transition coordinator, informational programming about health behaviors and coping, transfer planning, and meeting with peers, among other components. Though the authors found medium effect sizes for improved psychosocial health and treatment, the intervention did not affect treatment adherence. The authors posited that this may have been because they did not include an adherence intervention [43]. Regardless, these results are promising.

Some transition clinics studied were based solely on educational interventions [44, 45]. One study used an education-based intervention for pediatric heart transplant patients transferring to adult care [44]. The intervention utilized educational programming around topics related to health behaviors, transfer, and finances [44]. The program ended with a graduation ceremony and a gathering of clinic families. The proportion of calcineurin inhibitor levels outside the target range decreased significantly, suggesting a significant increase in adherence [44].

Recently, the possibility of providing virtual content has been explored [45]. Grady et al. [45] piloted a transition clinic for cardiac heart transplant patients transferring to adult care. The transition clinic included online modules with quizzes that patients completed after each module. Patients then discussed the module and subsequent quiz results with the pediatric heart transplant coordinator. Then, as in the treatment-as-usual arm, the adult heart transplant coordinator telephoned them at selected time points. Those in the intervention group received additional review of module content and factors related to the transfer. In contrast, the coordinator asked those in the control arm only whether they had any questions. The intervention had no significant effect on adherence [45]. The authors suggested that the lack of significant findings might be because of the study's small sample size, the lack of monitoring of attentiveness during the intervention, and high baseline adherence [45]. It is thus unclear whether similar interventions that include virtual content may be effective.

As a whole, this literature provides support for the use of transfer clinics to increase adherence rates. However, given the small sample sizes and retrospective methods of many of these studies, more research is needed to affirm their effectiveness. In addition, literature on integrating transitional strategies into standard care, particularly in IBD, appears to also be promising, but-to date-these studies have not included objective outcomes such as adherence [46, 47]. 


\subsection{Transition Coordinator}

Recent interventions, such as those just described, have included the role of a transition coordinator/navigator [48, 49]. The transition coordinator role is often performed by a mental health professional or healthcare worker who works with patients throughout the end of their pediatric care and through their transfer to adult services. Annunziato et al. [48] looked at the use of a transition coordinator in pediatric liver transplant patients to aid in the transfer to adult care. The transition coordinator was a licensed psychologist specializing in pediatric psychology whose work with patients spanned from at least 1 year before to 1 year after transfer. The coordinator helped patients with preparation for the transfer, served as a liaison between teams, helped families with care coordination and outreach, and developed outcome protocols. The control group had significantly increased rates of objective measures of nonadherence compared with the intervention group. The results of this study are promising and may suggest that the use of a transition coordinator who remains a constant throughout the process is most helpful [48].

A second study [49] further supported the use of a care coordinator who is familiar with the psychosocial and medical needs of patients during the transfer to adult care. This study assessed the effects of the use of a care coordinator and transfer clinic on transfer outcomes in patients with hemoglobinopathies. The care coordinator was a social worker, and their role was similar to that described in the study by Annunziato et al. [48]. The transfer clinic involved a meeting with both the pediatric and adult teams and the navigator regarding the transition, a tour of the adult facility, and provision of support resources. Self-reported adherence was significantly maintained or improved for patients in the intervention group compared with the control group. The transfer clinic also had a significant independent effect on adherence [49]. It appears that the role of a transition coordinator is an especially promising lower-scale intervention for improving adherence rates. As with the use of transfer clinics, more research is necessary in this area.

\subsection{Technology}

Despite the potential of the use of technology in increasing adherence outcomes during the transition period, research on its use remains limited [50,51] but is likely to grow given the current rise of telemedicine and telehealth interventions. The few studies that have addressed this topic have yielded mixed findings [50], and published materials frequently describe the methods rather than yet being able to share actual study results [52-55]. Two very recent comprehensive reviews concluded that, although adolescents and young adults were receptive to the technology, efficacy data were lacking $[51,56]$. Given the potential (although not yet realized) benefit of technological advances, we review some of the relevant studies here.

Some studies conducted in transition-age populations have used text message-based interventions [57-59]. One study looked at the use of text messages to increase adherence in a sample of pediatric liver transplant recipients of mixed ages (2-27 years) [58]. Messages were sent to either the patient or the care provider, reminding them to take or administer the medication. If the message was sent and the patient did not respond to confirm they took the medication, a subsequent message was sent to the caregiver. The patients had significantly improved adherence, as measured by the MLVI, and reduced rejection episodes. Though the study had a significant dropout rate $(33 \%)$ and a mean dropout time of 4 months, those who dropped out had adherence and rejection rates similar to completers. Similarly, Ting et al. [59] used a text message intervention in adolescents and young adults with childhood-onset systemic lupus erythematosus. One group of participants was assigned to treatment as usual, whereas the other group received a text message intervention. The intervention consisted of daily text message reminders and increased adherence to clinic visits but not to medication adherence, as compared with treatment as usual [59].

Text messages have also been utilized for HIV-positive adolescents and young adults [57]. Unlike the previously mentioned studies, the participants designed the text messages, which included both a reminder and an affirmation sent after they acknowledged taking their medication. The study compared adherence in an intervention and control group using both self-report and an objective measure of adherence (viral load, which was only available for some of the participants). The study found significant effects for the intervention using self-reported adherence, but these effects attenuated after 6 months, and there were no effects on viral load [57].

Another potential aid is phone applications (apps) [60, 61]. One study looked at the use of an app to improve diabetes self-management in young adults aged 14-22 years with poor glycemic control [60]. The app consisted of a chat room with peers, the ability to contact their health provider, information on carbohydrate counting, information about other related health behaviors (e.g., alcohol use), daily tips, a reminder function, and a section for parents on how they can support teens. As-treated analysis found no significant difference in $\mathrm{HbA} 1 \mathrm{c}$ between app users and the control group at 12 months. Participants used the app for a mean of only 10.5 days, so it may not have been useful or interesting enough to improve adherence [60]. A different app for diabetes self-management behaviors also yielded negative results [61]. The app included a reward system for more frequent blood glucose readings, more frequent 
in-range readings, and resolving any trends. Users could obtain iTunes gift cards from a reward system, and the app also identified trends in readings for users and asked them to determine likely causes. Only $35 \%$ of the sample was moderately or highly engaged in using the app over the 12 months of the study [61]. A different way to use technology is to use remote connection to a healthcare provider (similar to telehealth visits). This option has not been sufficiently studied, but a recent study showed a nonsignificant improvement in adherence in adolescent transplant recipients who participated in a video-enabled group intervention [62].

In conclusion, although technological solutions such as text messaging, various apps, and teleconferencing may be helpful in enhancing the management of transition of care, data regarding their efficacy are lacking. In fact, most of the few outcome-based quantitative studies that do exist have reported that such technologies are hard to use and ineffective. Our reading of the balance of the evidence at this juncture is that it points to a lack of efficacy. However, this can change, and robust controlled studies are urgently needed. Until we better understand those technologies (text messaging, remote conferencing, and various apps), our view is that their use to support the process of transition or transfer of care is not warranted outside of research studies to the exclusion of other evidence-based interventions. However, given the current coronavirus 2019 (COVID-19) pandemic, it is likely that technological solutions may be safer and more actionable for some patients. Thus, in the absence of any intervention or to make safer an existing intervention, technological solutions can be useful. However, we recommend that clinical teams utilize evidence-based interventions, such as transition coordinators, whenever possible.

Box 1 Key recommendations

Start transition planning early

Involve family members, if appropriate

Address psychosocial needs

Measure adherence throughout the process using

objective measures. Do not assume adherence

Provide education surrounding care, if necessary

Implement a transition coordinator

Utilize a transition coordinator

Revise planning as needed

\section{Limitations}

As stated, the current field of research on adherence during the transition period has many limitations. Most notably, small sample sizes and the sampling of patients who were initially adherent to medication limit the conclusions of current research [12]. Additionally, many studies utilized self-report adherence measures, which may have overestimated actual adherence [12,63]. This is also the case for studies that utilized measures of adherence focused on behaviors such as logbooks or pharmacy refill data. This problem has been clearly noted in the literature related to adherence in asthma [64], diabetes [65], transplant [12], and IBD [66] populations, among others. Adherence may also change over time, limiting the testability of the longterm effects of adherence interventions [14]. Changing levels of family involvement in medication management may also complicate sustainability of results if interventions only include the patient. Lastly, sample sizes in adherence research are often small and non-diverse, which may also limit generalizability.

\section{Conclusions}

Future studies assessing adherence should try to address these flaws. With these caveats, we make some commonsense recommendations. First, the transfer to adult care is a process. Planning that meaningfully includes caregivers should precede it. Second, providers should measure adherence using objective measures throughout the treatment process to address any issues that arise early on. Third, it is essential that mental healthcare needs are addressed during the transition period. Fourth, implementing programs such as a transition coordinator/navigator is likely helpful, especially if the point person remains with patients throughout the process. Fifth, a transition clinic that both integrates educational interventions and addresses psychosocial needs may also be an effective, albeit "scaled-up," approach. Given the lack of research on the use of technology, it is difficult to make any firm recommendations on its use. Studies on the use of technology in increasing adherence are likely to continue to proliferate, especially in response to COVID19. Finally, research that provides insights into the transition experience for diverse and/or traditionally underserved patients is lacking. Data from larger, more heterogeneous samples are needed to inform interventions before one-sizefits-all strategies are routinely adopted.

\section{Declarations}

Funding The authors are funded by the National Institutes of Health \#R01 DK080740.

Conflict of Interest Bianca R. Campagna, Kristen Weatherley, Eyal Shemesh, and Rachel A. Annunziato have no conflicts of interest that are directly relevant to the content of this article.

Availability of data and material Not applicable. 
Ethics approval Not applicable.

Consent Not applicable.

Author contributions All authors contributed to study conception and design. The first draft of the manuscript was written by BC and was critically revised by KW, ES, and RA. All authors read and approved the final manuscript.

\section{References}

1. White PH, Cooley WC, Transitions Clinical Report Authoring Group, American Academy of Pediatrics, American Academy of Family Physicians, American College of Physicians. Supporting the health care transition from adolescence to adulthood in the medical home. Pediatrics. 2018;142:e20182587.

2. Fegran L, Hall EOC, Uhrenfeldt L, Aagaard H, Ludvigsen MS. Adolescents' and young adults' transition experiences when transferring from paediatric to adult care: a qualitative metasynthesis. Int J Nurs Stud. 2014;51:123-35.

3. Bemrich-Stolz CJ, Halanych JH, Howard TH, Hilliard LM, Lebensburger JD. Exploring adult care experiences and barriers to transition in adult patients with sickle cell disease. Int J Hematol Ther. NIH Public Access; 2015;1.

4. Philbin MM, Tanner AE, Chambers BD, Ma A, Ware S, Lee S, et al. Transitioning HIV-infected adolescents to adult care at 14 clinics across the United States: using adolescent and adult providers' insights to create multi-level solutions to address transition barriers. AIDS Care. 2017;29:1227-344.

5. Wiener LS, Kohrt B-A, Battles HB, Pao M. The HIV experience: youth identified barriers for transitioning from pediatric to adult care. J Pediatr Psychol. 2011;36:141-54.

6. Lebrun-Harris LA, McManus MA, Ilango SM, Cyr M, McLellan SB, Mann MY, et al. Transition planning among US youth with and without special health care needs. Pediatrics. 2018;142:e20180194.

7. Kahana SY, Frazier TW, Drotar D. Preliminary quantitative investigation of predictors of treatment non-adherence in pediatric transplantation: a brief report. Pediatr Transplant. 2008;12:656-60.

8. Watson AR. Non-compliance and transfer from pediatric to adult transplant unit. Pediatr Nephrol. 2000;14:0469-472.

9. Annunziato RA, Emre S, Shneider B, Barton C, Dugan CA, Shemesh E. Adherence and medical outcomes in pediatric liver transplant recipients who transition to adult services: changes in adherence after transition. Pediatr Transplant. 2007;11:608-14.

10. Gabriel P, McManus M, Rogers K, White P. Outcome evidence for structured pediatric to adult health care transition interventions: a systematic review. J Pediatr. 2017;188(263-269):e15.

11. Campbell F, Biggs K, Aldiss SK, O’Neill PM, Clowes M, McDonagh J, et al. Transition of care for adolescents from pediatric services to adult health services. Cochrane Effective Practice and Organisation of Care Group, editor. Cochrane Database Syst Rev [Internet]. 2016. https://doi.org/10.1002/14651858.CD009794. pub2.

12. Duncan S, Annunziato RA, Dunphy C, LaPointe RD, Shneider BL, Shemesh E. A systematic review of immunosuppressant adherence interventions in transplant recipients: decoding the streetlight effect. Pediatr Transplant. 2018;22:e13086.

13. Supelana C, Annunziato RA, Schiano TD, Anand R, Vaidya $\mathrm{S}$, Chuang K, et al. Medication level variability index predicts rejection, possibly due to nonadherence, in adult liver transplant recipients: assessing nonadherance after transplantation. Liver Transpl. 2014;20:1168-77.

14. Shemesh E, Duncan S, Anand R, Shneider BL, Alonso EM, Mazariegos GV, et al. Trajectory of adherence behavior in pediatric and adolescent liver transplant recipients: the medication adherence in children who had a liver transplant cohort: Shemesh et al. Liver Transpl. 2018;24:80-8.

15. Fenton N, Ferris M, Ko Z, Javalkar K, Hooper SR. The relationship of health care transition readiness to disease-related characteristics, psychosocial factors, and health care outcomes: preliminary findings in adolescents with chronic kidney disease. J Pediatr Rehabil Med. 2015;8:13-22.

16. Fishman LN, Ziniel SI, Adrichem ME, Fernandes SM, Arnold J. Provider awareness alone does not improve transition readiness skills in adolescent patients with inflammatory bowel disease. J Pediatr Gastroenterol Nutr. 2014;59:221-4.

17. Fredericks EM, Dore-Stites D, Well A, Magee JC, Freed GL, Shieck V, et al. Assessment of transition readiness skills and adherence in pediatric liver transplant recipients: transition and adherence. Pediatr Transplant. 2010;14:944-53.

18. Rosen D, Annunziato R, Colombel JF, Dubinsky M, Benkov $\mathrm{K}$. Transition of inflammatory bowel disease care: assessment of transition readiness factors and disease outcomes in a young adult population. Inflamm Bowel Dis. 2016;22:702-8.

19. Jones MR, Frey SM, Riekert K, Fagnano M, Halterman JS. Transition readiness for talking with providers in urban youth with asthma: associations with medication management. J Adolesc Health. 2019;64:265-71.

20. Verma T, Rohan J. Examination of transition readiness, medication adherence, and resilience in pediatric chronic illness populations: a pilot study. Int J Environ Res Public Health. 2020;17:1905.

21. Annunziato RA, Stuber ML, Supelana CJ, Dunphy C, Anand R, Erinjeri J, et al. The impact of caregiver post-traumatic stress and depressive symptoms on pediatric transplant outcomes. Pediatr Transplant [Internet]. 2020. https://doi.org/10.1111/petr.13642.

22. Substance Abuse and Mental Health Services Administration. Mental Health Services Administration (2018). Key substance use and mental health indicators in the United States: Results from the 2017 National Survey on Drug Use and Health (HHS Publication No. SMA 18-5068, NSDUH Series H-53). Rockville, MD: Center for Behavioral Health Statistics and Quality. Subst Abuse Ment Health Serv Adm [Internet]. 2019; Available from: https:// www.samhsa.gov/data. Accessed 2020 May 2.

23. Blanco C, Okuda M, Wright C, Hasin DS, Grant BF, Liu S-M, et al. Mental health of college students and their non-collegeattending peers: results from the national epidemiologic study on alcohol and related conditions. Arch Gen Psychiatry. 2008;65:1429.

24. DiMatteo MR, Lepper HS, Croghan TW. Depression is a risk factor for noncompliance with medical treatment: meta-analysis of the effects of anxiety and depression on patient adherence. Arch Intern Med. 2000;160:2101.

25. Annunziato RA, Arrato N, Rubes M, Arnon R. The importance of mental health monitoring during transfer to adult care settings as examined among pediatric transplant recipients: mental health and transfer from pediatrics. J Paediatr Child Health. 2015;51:220-2.

26. Killian MO, Schuman DL, Mayersohn GS, Triplett KN. Psychosocial predictors of medication non-adherence in pediatric organ transplantation: a systematic review. Pediatr Transplant. 2018;22:e13188.

27. Taggart Wasson L, Shaffer JA, Edmondson D, Bring R, Brondolo E, Falzon L, et al. Posttraumatic stress disorder and nonadherence to medications prescribed for chronic medical conditions: a metaanalysis. J Psychiatr Res. 2018;102:102-9. 
28. Supelana C, Annunziato RA, Kaplan D, Helcer J, Stuber ML, Shemesh E. PTSD in solid organ transplant recipients: current understanding and future implications. Pediatr Transplant. 2016;20:23-33.

29. Shemesh E, Rudnick A, Kaluski E, Milovanov O, Salah A, Alon $\mathrm{D}$, et al. A prospective study of posttraumatic stress symptoms and nonadherence in survivors of a myocardial infarction (MI). Gen Hosp Psychiatry. 2001;23:215-22.

30. Husain SA, Edmondson D, Kautz M, Umland R, Kronish IM. Posttraumatic stress disorder due to acute cardiac events and aversive cognitions towards cardiovascular medications. J Behav Med. 2018;41:261-8.

31. Shemesh E, Lurie S, Stuber ML, Emre S, Patel Y, Vohra P, et al. A pilot study of posttraumatic stress and nonadherence in pediatric liver transplant recipients. Pediatrics. 2000;105:e29-e2929.

32. Lewis SJ, Arseneault L, Caspi A, Fisher HL, Matthews T, Moffitt TE, et al. The epidemiology of trauma and post-traumatic stress disorder in a representative cohort of young people in England and Wales. Lancet Psychiatry. 2019;6:247-56.

33. Cotter G, Milo-Cotter O, Rubinstein D, Shemesh E. Posttraumatic stress disorder: a missed link between psychiatric and cardiovascular morbidity? CNS Spectr. 2006;11:129-36.

34. Shemesh E, Annunziato RA, Weatherley BD, Cotter G, Feaganes JR, Santra M, et al. A randomized controlled trial of the safety and promise of cognitive-behavioral therapy using imaginal exposure in patients with posttraumatic stress disorder resulting from cardiovascular illness. J Clin Psychiatry. 2011;72:168-74.

35. Psihogios AM, Fellmeth H, Schwartz LA, Barakat LP. Family functioning and medical adherence across children and adolescents with chronic health conditions: a meta-analysis. J Pediatr Psychol. 2019;44:84-97.

36. Iskander JM, Rohan JM, Pendley JS, Delamater A, Drotar D. A 3-year prospective study of parent-child communication in early adolescents with type 1 diabetes: relationship to adherence and glycemic control. J Pediatr Psychol. 2015;40:109-20.

37. Reed-Knight B, Blount RL, Gilleland J. The transition of health care responsibility from parents to youth diagnosed with chronic illness: a developmental systems perspective. Fam Syst Health. 2014;32:219-34.

38. Wysocki T, Harris MA, Buckloh LM, Mertlich D, Lochrie AS, Mauras N, et al. Randomized trial of behavioral family systems therapy for diabetes: maintenance of effects on diabetes outcomes in adolescents. Diabetes Care. 2007;30:555-60.

39. Duncan CL, Hogan MB, Tien KJ, Graves MM, Chorney JM, Zettler MD, et al. Efficacy of a parent-youth teamwork intervention to promote adherence in pediatric asthma. J Pediatr Psychol. 2013;38:617-28

40. Cole R, Ashok D, Razack A, Azaz A, Sebastian S. Evaluation of outcomes in adolescent inflammatory bowel disease patients following transfer from pediatric to adult health care services: case for transition. J Adolesc Health. 2015;57:212-7.

41. McQuillan RF, Toulany A, Kaufman M, Schiff JR. Benefits of a transfer clinic in adolescent and young adult kidney transplant patients. Can J Kidney Health Dis. 2015;2:81

42. Agarwal S, Raymond JK, Schutta MH, Cardillo S, Miller VA, Long JA. An adult health care-based pediatric to adult transition program for emerging adults with type 1 diabetes. Diabetes Educ. 2017;43:87-96.

43. Hilderson D, Moons P, Van der Elst K, Luyckx K, Wouters C, Westhovens R. The clinical impact of a brief transition programme for young people with juvenile idiopathic arthritis: results of the DON'T RETARD project. Rheumatology. 2016;55:133-42.

44. Anton CM, Anton K, Butts RJ. Preparing for transition: the effects of a structured transition program on adolescent heart transplant patients' adherence and transplant knowledge. Pediatr Transplant. 2019;23:e13544
45. Grady KL, Andrei A-C, Shankel T, Chinnock R, Miyamoto SD, Ambardekar AV, et al. Pediatric heart transplantation: transitioning to adult care (TRANSIT): feasibility of a pilot randomized controlled trial. J Card Fail. 2019;25:948-58.

46. Yerushalmy-Feler A, Ron Y, Barnea E, Nachum A, Matalon S, Dali-levy M, et al. Adolescent transition clinic in inflammatory bowel disease: quantitative assessment of self-efficacy skills. Eur J Gastroenterol Hepatol. 2017;29:831-7.

47. Fu N, Jacobson K, Round A, Evans K, Qian H, Bressler B. Transition clinic attendance is associated with improved beliefs and attitudes toward medicine in patients with inflammatory bowel disease. World J Gastroenterol. 2017;23:5405.

48. Annunziato RA, Baisley MC, Arrato N, Barton C, Henderling F, Arnon R, et al. Strangers headed to a strange land? A pilot study of using a transition coordinator to improve transfer from pediatric to adult services. J Pediatr. 2013;163:1628-33.

49. Allemang B, Allan K, Johnson C, Cheong M, Cheung P, Odame $\mathrm{I}$, et al. Impact of a transition program with navigator on loss to follow-up, medication adherence, and appointment attendance in hemoglobinopathies. Pediatr Blood Cancer. 2019;66:e27781.

50. Badawy SM, Thompson AA, Kuhns LM. Medication adherence and technology-based interventions for adolescents with chronic health conditions: a few key considerations. JMIR MHealth UHealth. 2017;5:e202.

51. Virella Pérez YI, Medlow S, Ho J, Steinbeck K. Mobile and web-based apps that support self-management and transition in young people with chronic illness: systematic review. J Med Internet Res. 2019;21:e13579.

52. Holtz BE, Mitchell KM, Hershey DD, Cotten SR, Holmstrom AJ, Richman J, et al. Using an mHealth App to transition care of type 1 diabetes from parents to teens: protocol for a pilot study. JMIR Res Protoc. 2018;7:e10803.

53. Lopez KN, O'Connor M, King J, Alexander J, Challman M, Lovick DK, et al. Improving transitions of care for young adults with congenital heart disease: mobile app development using formative research. JMIR Form Res. 2018;2:e16.

54. Curtis K, Lebedev A, Aguirre E, Lobitz S. A medication adherence app for children with sickle cell disease: qualitative study. JMIR MHealth UHealth. 2019;7:e8130.

55. Holtz BE, Murray KM, Hershey DD, Richman J, Dunneback JK, Vyas A, et al. The design and development of MyT1DHero: a mobile app for adolescents with type 1 diabetes and their parents. J Telemed Telecare. 2019;25:172-80.

56. Low JK, Manias E. Use of technology-based tools to support adolescents and young adults with chronic disease: systematic review and meta-analysis. JMIR MHealth UHealth. 2019; 7:e12042

57. Garofalo R, Kuhns LM, Hotton A, Johnson A, Muldoon A, Rice D. A randomized controlled trial of personalized text message reminders to promote medication adherence among HIV-positive adolescents and young adults. AIDS Behav. 2016;20:1049-59.

58. Miloh T, Annunziato R, Arnon R, Warshaw J, Parkar S, Suchy FJ, et al. Improved adherence and outcomes for pediatric liver transplant recipients by using text messaging. Pediatrics. 2009; 124:e844-e850850.

59. Ting TV, Kudalkar D, Nelson S, Cortina S, Pendl J, Budhani S, et al. Usefulness of cellular text messaging for improving adherence among adolescents and young adults with systemic lupus erythematosus. J Rheumatol. 2012;39:174-9.

60. Castensøe-Seidenfaden P, Husted GR, Jensen AK, Hommel E, Olsen B, Pedersen-Bjergaard U, et al. Testing a Smartphone App (Young with Diabetes) to improve self-management of diabetes over 12 months: randomized controlled trial. JMIR MHealth UHealth. 2018;6:e141.

61. Goyal S, Nunn CA, Rotondi M, Couperthwaite AB, Reiser S, Simone A, et al. A Mobile App for the self-management of type 1 
diabetes among adolescents: a randomized controlled trial. JMIR MHealth UHealth. 2017;5:e82.

62. Kelly SL, Steinberg EA, Suplee A, Upshaw NC, Campbell KR, Thomas JF, et al. Implementing a home-based Telehealth Group adherence intervention with adolescent transplant recipients. Telemed E Health. 2019;25:1040-8.

63. Stirratt MJ, Dunbar-Jacob J, Crane HM, Simoni JM, Czajkowski S, Hilliard ME, et al. Self-report measures of medication adherence behavior: recommendations on optimal use. Transl Behav Med. 2015;5:470-82.
64. Desai M, Oppenheimer JJ. Medication adherence in the asthmatic child and adolescent. Curr Allergy Asthma Rep. 2011;11:454-64.

65. Blackwell M, Wheeler BJ. Clinical review: the misreporting of logbook, download, and verbal self-measured blood glucose in adults and children with type I diabetes. Acta Diabetol. 2017;54:1-8.

66. Hommel KA, Davis CM, Baldassano RN. Objective versus subjective assessment of oral medication adherence in pediatric inflammatory bowel disease. Inflamm Bowel Dis. 2009;15:589-93. 